\title{
Understanding Constipation: A Cross-sectional Study from a Developing Country Setting
}

\author{
Minaz Mawani1, Abdul Azeem³, Sakina Gheewala33, Nazish Butt² and Shahab Abid 1
}

\begin{abstract}
A cross-sectional study was conducted in the Gastroenterology Clinics of The Aga Khan Hospital and Jinnah Postgraduate Medical Centre in Karachi. All patients complaining of constipation were included. Patients with alarm features were excluded. Interviews were conducted by trained doctors using a structured Rome III based pre-tested questionnaire and Bristol Stool Form Scale. Out of 396 participants, $186(47.0 \%)$ had FC and $209(52.7 \%)$ had IBS-C. Based upon Bristol Stool Form Scale, only $214(54 \%)$ reported to have slow transit constipation (BSS $\leq 2)$. A higher proportion of women reported severe form of constipation as compared to men $(81.5 \%, n=150$ vs. $65.1 \%, n=138 p<0.001)$. However, a higher percentage of men reported using medications to treat their symptoms $(71.2 \%, n=151$ vs. $57.1 \%, n=105 p=0.003)$. Proper understanding of patient's symptoms is essential for optimizing individual patient management.
\end{abstract}

Key Words: Constipation, Irritable bowel syndrome, Karachi, Functional constipation.

Constipation is the most frequently reported functional gastrointestinal disorder associated with reduced quality of life and huge economic burden. Prevalence in general adult population has been reported to range from $2.5 \%$ to $79 \%,{ }^{1}$ with a high amount of variability due to different definitions being used. Patients usually define constipation in terms of function such as straining, difficulty passing stool etc. In contrast, physicians would generally define it as having infrequent bowel movements. Due to this discordance, about half of the patients remain dissatisfied with the treatment. ${ }^{2}$ There is a lack of data on how patients understand constipation. Therefore, it was intended to explore perceptions about constipation among patients presenting to gastrointestinal clinics in Karachi, Pakistan.

A cross-sectional study was conducted at the Gastroenterology Outpatient Clinics of The Aga Khan Hospital and Jinnah Postgraduate Medical Centre. Purposive sampling was done and all patients who reported to have constipation were included. Patients with alarm features such as weight loss and gastrointestinal bleeding were excluded.

A questionnaire was developed including questions on general information, constipation symptoms, Bristol stool form scale (BSS) and standard ROME III diagnostic criteria for functional constipation (FC) and irritable bowel

1 Department of Medicine / Gastrology2, The Aga Khan University Hospital, Karachi, Pakistan

3 Medical Student, The Aga Khan University Hospital, Karachi, Pakistan

Correspondence: Prof. Shahab Abid, Department of Medicine, The Aga Khan University Hospital, Stadium Road, Karachi, Pakistan

E-mail: shahab.abid@aku.edu

Received: March 22, 2018; Accepted: October 24, 2018 syndrome-constipation (IBS-C). ${ }^{3}$ It was administered by trained doctors.

Continuous variable such as age was reported as mean and standard deviation. Frequencies with percentages were reported for all categorical variables such as gender. Characteristics of patients who were found to have FC were compared with those having IBS-C using either independent sample T-test or ANOVA for quantitative variables and Chi-square test for categorical variables. P-value $<0.05$ was considered as significant. All analyses were done using SPSS (statistical package for social scientists) version 19.

The study was approved by The Aga Khan University Ethics Review Committee and heads of department in both the study centres. Written informed consent was obtained from the participants.

Out of 396 patients with age between 15 and 80 years, $212(53.5 \%)$ were males. About $47.2 \%$ (187) of respondents were $\leq 35$ years, $37.9 \%$ (150) were between 36 and 55 years, $9.3 \%$ (37) were between 56 and 65 years, whereas only $5.6 \%$ (22) were between 66 and 80 years of age. A higher percentage $(43.2 \%, n=171)$ of patients reported having moderate constipation. Out of all, $47 \%$ ( $n=186$ ) fulfilled the criteria for FC according to ROME III of which 97 (52.2\%) were classified as having constipation on both ROME III and BSS. Almost half of the participants $(n=209,52.7 \%)$ were categorized as having IBS-C of which $117(56.0 \%)$ had constipation according to both ROME III and BSS. These differences, however, were not found to be statistically significant $(p=0.45)$. Overall, only $214(54 \%)$ were classified as having constipation based on BSS.

Significantly, higher number of females reported greater severity of constipation as compared to males $(81.5 \%$, $\mathrm{n}=150$ vs. $65.1 \%, \mathrm{n}=138, \mathrm{p}<0.001)$. However, a higher 


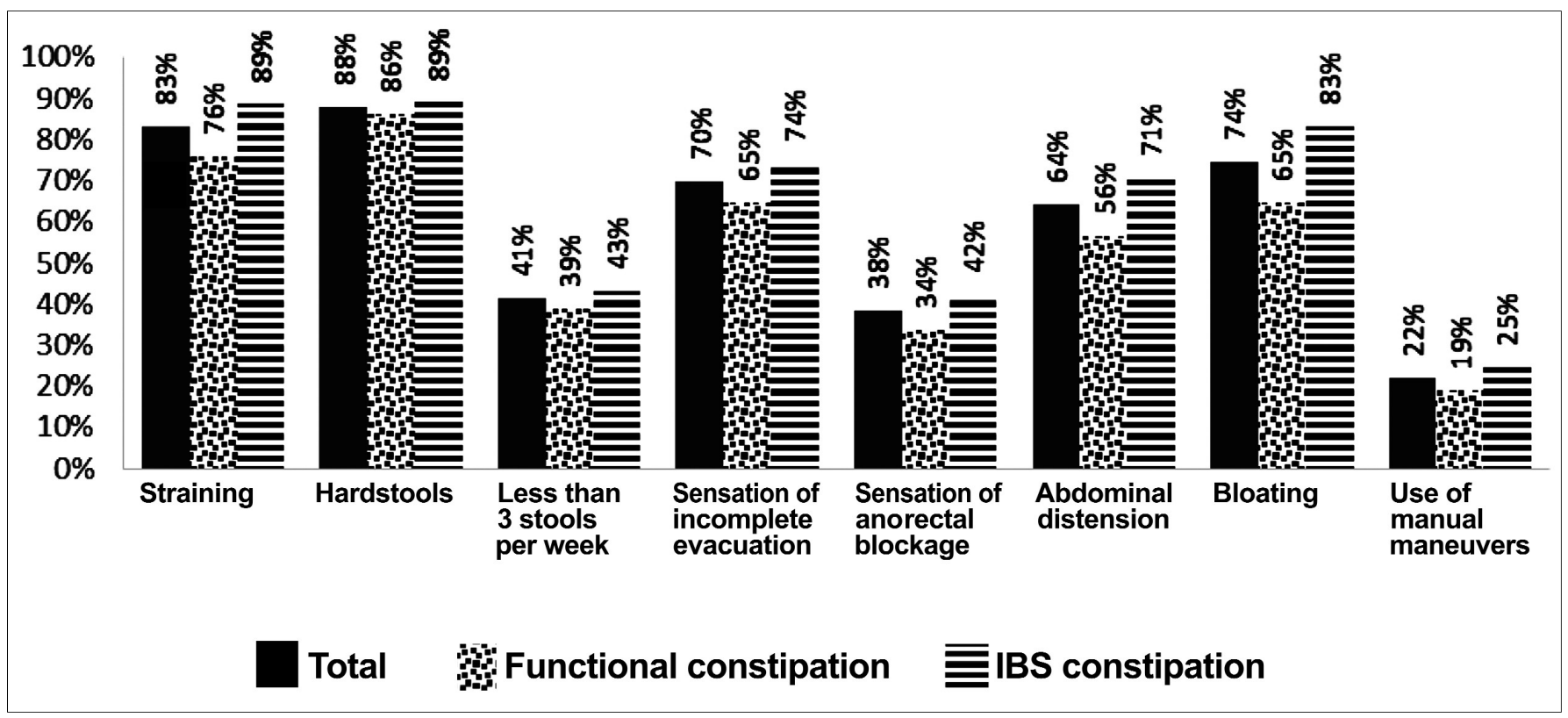

Figure 1: Comparison of signs and symptoms of patients with irritable bowel syndrome-constipation and functional-constipation.

percentage of males reported using any kind of laxatives $(71.2 \%, n=151$ vs. $57.1 \%, n=105, p=0.003)$. A significantly higher number of females reported the symptoms of distension and having less than 3 stools per week, but

\begin{tabular}{|c|c|c|c|}
\hline Characteristics & $\begin{array}{c}\text { Male } \\
\mathrm{n}(\%) \\
\mathrm{n}=212\end{array}$ & $\begin{array}{c}\text { Female } \\
\mathrm{n}(\%) \\
\mathrm{n}=184\end{array}$ & $\mathrm{p}$-values \\
\hline \multicolumn{3}{|c|}{ Constipation severity } & 0.001 \\
\hline Mild & $74(34.9)$ & $34(18.5)$ & \\
\hline Moderate & $84(39.6)$ & $87(47.3)$ & \\
\hline Severe & $54(25.5)$ & $63(34.2)$ & \\
\hline \multicolumn{3}{|l|}{ Medications } & 0.003 \\
\hline Any medication & $151(71.2)$ & $105(57.1)$ & \\
\hline No medication & $61(28.8)$ & 79 (42.9) & \\
\hline \multicolumn{3}{|c|}{ Less than 3 stools per week } & 0.001 \\
\hline Yes & $71(33.8)$ & $91(49.7)$ & \\
\hline No & $139(66.2)$ & $92(50.3)$ & \\
\hline \multicolumn{3}{|c|}{ Sensation of incomplete evacuation } & 0.33 \\
\hline Yes & $152(71.7)$ & $123(67.2)$ & \\
\hline No & $60(28.3)$ & $60(32.8)$ & \\
\hline \multicolumn{3}{|c|}{ Sensation of anorectal blockage } & 0.02 \\
\hline Yes & $92(43.6)$ & $59(32.4)$ & \\
\hline No & $119(56.4)$ & $123(67.6)$ & \\
\hline \multicolumn{3}{|c|}{ Abdominal distension } & 0.01 \\
\hline Yes & $123(58.6)$ & $130(70.7)$ & \\
\hline No & $87(41.4)$ & $54(29.3)$ & \\
\hline \multicolumn{3}{|l|}{ Bloating } & 0.31 \\
\hline Yes & $152(72.7)$ & $142(77.2)$ & \\
\hline No & $57(27.3)$ & $42(22.8)$ & \\
\hline \multicolumn{3}{|c|}{ Use of manual maneuvers } & 0.49 \\
\hline Yes & $44(20.9)$ & $43(23.8)$ & \\
\hline No & $167(79.1)$ & $138(76.2)$ & \\
\hline \multicolumn{3}{|l|}{ Bristol scoring } & 0.03 \\
\hline Constipation & $125(59.2)$ & $89(48.4)$ & \\
\hline Others & $86(40.8)$ & $95(51.6)$ & \\
\hline
\end{tabular}

the frequency of anorectal blockage was most commonly reported by males (Table I).

A comparison of patients' characteristics between FC and IBS-C is given in Figure 1. A higher number of males had FC $(58.3 \%, n=123)$, whereas a higher number of females had IBS-C $(65.8 \%, n=121)$. Participants with IBS-C complained of severe constipation $(35.4 \%, n=74$ vs. $23.1 \%, n=43, p=0.016$ ). No significant differences were observed in Bristol stool form scale scoring amongst the two groups $(p=0.44)$.

Patients were also inquired about the modalities that they used for treating their constipation. About $60 \%$ $(n=238)$ patients reported using one type of remedy, whereas $4.5 \% \quad(n=18)$ of patients reported using a combination of two or more modalities, which were use of fibre, and purgatives. Patients reported using fibres, purgatives, home remedies, non-specific medications, Hakimi/homeopathic medications and local applicants. Use of medications increased with increasing age (ANOVA, $p=0.002$ ). Number of remedies did not vary significantly with the variant of constipation.

Literature reports an overlap between functionalconstipation and irritable bowel syndrome-constipation. ${ }^{4}$ In the present series, out of all the participants reporting to have constipation, only $46.9 \%$ fulfilled the ROME III criteria for FC, whereas another study from Karachi reported $53 \%$ of patients fulfilling the ROME III criteria for FC. ${ }^{5}$ Therefore, one should remain vigilant in diagnosing since treatment that works best for functional-constipation will not be as good for the treatment of IBS-C constipation.

Interestingly about half of the participants reporting IBS-C and FC on ROME III criteria did not have constipation according to Bristol stool form scale in our series. Since 
these responses were based on one-time questioning, the reported responses might be different from the actual. Based on the assumption that the reported scores are correct representation of the actual scenario then half of the participants had a normal transit constipation.

In the present paper, a higher percentage of females reported severe constipation. However, more males reported using any kind of treatment. This could be due to higher health seeking behaviour amongst males. In contrast, another study reported an equal male to female ratio in terms of constipation, but not addressing severity. ${ }^{5}$ This also signifies social and cultural aspects of a male dominant society where males enjoy a superior position and, therefore, receive more healthcare for their problems. Similar findings have been reported from India and other Asian countries. ${ }^{6}$

This study had certain limitations. First, it was based on subjective reporting of symptoms by patients and did not include any radiological or motility assessments which are recommended. It implies that this study sample might have included a subset of patients with dyssynergic defecation which could not be diagnosed in the absence of anorectal manometry. Secondly, these patients could not be followed to see if their condition improved in response to treatment or not. Third, we did not assess quality of life amongst these patients.
Almost half of the patients were not suffering from slow transit constipation. A large number of patients had diverse accompanying defecatory symptoms. Therefore, treatment regimens reducing transit time are not appropriate for these patients. Proper understanding of patient's perspective of constipation is essential for optimizing management.

\section{REFERENCES}

1. Mugie SM, Benninga MA, Di Lorenzo C. Epidemiology of constipation in children and adults: a systematic review. Best Pract Res Clin Gastroenterol 2011; 25:3-18.

2. Johanson J, Kralstein J. Chronic constipation: a survey of the patient perspective. Aliment Pharmacol Ther 2007; 25:599-608.

3. ROME III: The Functional Gastrointestinal Disorders. Drossman DA, editor. McLean, Virginia 2006

4. Wong RK, Palsson OS, Turner MJ, Levy R, Feld AD, von Korff M, et al. Inability of the Rome III criteria to distinguish functional constipation from constipation subtype irritable bowel syndrome. Am J Gastroenterol 2010; 105:2228-34

5. Khatri PK, Ali AD, Alzadjali N, Bhagia G, Khaliqdina SJ, Aziz S. Frequency of functional constipation in 3 different populations and its causative factors. J Pak Med Assoc 2011; 61:1149-52.

6. Jain A, Gupta O, Jajoo U, Sidhwa H. Clinical profile of irritable bowel syndrome at a rural-based teaching hospital in central India. J Assoc Physicians India 1991; 39:385-6. 